PESQUIMAT 24(2): $82-96$ (2021)

ISSN:1560-912X/ ISSN-E:1609-8439

https://doi.org/10.15381/pesquimat.v24i2.21869

Facultad de Ciencias Matemáticas - UNMSM

\title{
Análisis de Estabilidad Local de la Dinámica COVID-19 de un Modelo SIR con Tasas de Transmisión no Lineal
}

\author{
Javier Huapaya Quispe ${ }^{1}$, Willy Barahona Martíne $2^{2}$, Rocío De La Cruz Marcacuzco ${ }^{3}$
}

Resumen: En este artículo, consideramos un modelo SIR modificado, implementando una población de Patógenos que interactúa con una población humana de Susceptibles, con lo cual tendremos en nuestro sistema 4 ecuaciones diferenciales ordinarias.

El objetivo de este trabajo, es analizar la estabilidad del punto libre de enfermedad (local y global) y el punto de equilibrio endémico (local) de este modelo matemático. Además se presentan simulaciones numéricas al modelo para contrastar los efectos de las tasas de transmisión no lineal y otros parámetros.

Palabras claves: Modelo SIR, Estabilidad, Número Básico de Reproducción, Criterio de Routh Hurwitz, Matriz de la Próxima Generación, Simulación Numérica.

\section{Local Stability Analysis of COVID-19 Dynamics of a SIR Model with Non-Linear Transmission Rates}

\begin{abstract}
In this paper, we consider a modified SIR model, implementing a population of Pathogens interacting with a human population of Susceptibles, with which we will have 4 ordinary differential equations in our system. The objective of this work is to analyze the stability of the disease free point (local and global) and the endemic equilibrium point (local) of this mathematical model. In addition, numerical simulations to the model are presented to contrast the effects of nonlinear transmission rates and other parameters.
\end{abstract}

Keywords: SIR model, Stability, Basic Reproduction Number, Routh Hurwitz Criterion, Next Generation Matrix, Numerical Simulation.

Recibido: 15/11/2021. Aceptado: 18/12/2021. Publicado online: 30/12/2021.

\footnotetext{
(C) Los autores. Este artículo es publicado por la Revista PESQUIMAT de la Facultad de Ciencias Matemáticas, Universidad Nacional Mayor de San Marcos. Este es un artículo de acceso abierto, distribuido bajo los términos de la licencia Creative Commons Atribucion-No Comercia-CompartirIgual 4.0 Internacional.(http://creativecommons.org/licenses/by-nc-sa/4.0/) que permite el uso no comercial, distribución y reproducción en cualquier medio, siempre que la obra original sea debidamente citada. Para uso comercial, por favor póngase en contacto y reproducción en cualquier medio, siempre que

${ }^{1}$ UNMSM, Facultad de Ciencias Matemáticas, e-mail: javier.huapaya2@unmsm.edu.pe

${ }^{2}$ UNMSM, Facultad de Ciencias Matemáticas. e-mail: wbarahonam@unmsm.edu.pe

${ }^{3}$ UNMSM, Facultad de Ciencias Matemáticas, e-mail: rdelacruzm@unmsm.edu.pe
} 


\section{Introducción}

La Epidemiología Matemática es la encargada de estudiar la propagación de enfermedades, que tiene su origen a partir del modelo propuesto en 1927 por Kermarck y McKendrik [1], modelo SIR, que divide a la población en tres compartimentos: Susceptibles, Infectados y Recuperados.

\section{Preliminares}

El principio invarianza de LaSalle, es una herramienta muy útil que a la par del Teorema de Lyapunov, estudian la estabilidad en los puntos de equilibrio. Sin embargo los teoremas de Liapunov al ser difícil de encontrar la función candidata, se recurre a este principio para debilitar algunas condiciones del Teorema de Lyapunov [2, ,3]. Consideremos la ecuación diferencial

$$
\left\{\begin{aligned}
x^{\prime}(t) & =f(x) \\
x(0) & =x_{0}
\end{aligned}\right.
$$

Definición 2.1 El conjunto de todos los puntos límites positivos de $x(t)$ se llama conjunto límite positivo de $x(t)$, y es denotado por $L^{+}$.

Definición 2.2 $\mathcal{M}$ será un conjunto positivamente invariante de (1), si

$$
x(T) \in \mathcal{M} \Longrightarrow x(t) \in \mathcal{M}, \forall t>T
$$

Definición 2.3 Un conjunto $\mathcal{M}$ es asintóticamente atractivo, si

$$
x(t) \rightarrow \mathcal{M} \text {, cuando } t \rightarrow \infty
$$

Lema 2.4 Si una solución $x(t)$ de (1) es acotada y se encuentra siempre en su dominio $\mathcal{D} \subseteq \mathbb{R}^{n}$, $\forall t \geq 0$, entonces su conjunto límite positivo $L^{+}$es un conjunto invariante, no vacío y compacto.

Teorema 2.5 Sea $\Omega \subset \mathcal{D}$ compacto tal que es positivamente invariante con respecto a (1). Sea $V: \mathcal{D} \rightarrow \mathbb{R}$ una función continuamente diferenciable tal que $V^{\prime} \leq 0$ en $\Omega$. Sea $E$ el conjunto de todos los puntos de $\Omega$ donde $V^{\prime}=0$. Sea $\mathcal{M}$ el mayor conjunto invariante contenido en $E$. Entonces, toda solución que inicia en $\Omega$ se aproxima a $\mathcal{M}$ cuando $t \rightarrow \infty$.

Demostración. Ver [3] ,pag 128.

\section{Resultado Principal}

En este artículo estudiaremos la dinámica de un modelo SIR modificado con tasa de incidencia no lineal, adicionando el contagio a través del contacto con una superficie contaminada o por aerosoles ocasionada por un patógeno. Nuestro trabajo consistirá en el análisis de estabilidad de los puntos de equilibrio y realizar algunas simulaciones numéricas al modelo planteado.

\subsection{Modelo SIR-P}

En este estudio, se pretende modelar la propagación y transmisión de una enfermedad infecciosa, considerando dos poblaciones que interactuan entre sí, la población humana y el patógeno, en el caso de la población humana es la que hospeda al patógeno.

$$
N(t)=S(t)+I(t)+R(t)
$$

En este modelo consideraremos dos formas de contagios: 
- De humano infectado a humano susceptible, y

- Del medio ambiente a humano susceptible .

Donde las tasas de transmisión de contagio son respectivamente $\frac{\beta_{1} S I}{k_{1}+I}$ y $\frac{\beta_{2} S P}{k_{2}+P}$.

Dado que se sabe que el patógeno $P(t)$ sobrevive en el medio ambiente durante algunos días, es probable que los individuos susceptibles $S(t)$ en contacto cercano con este medio se expongan a estos patógenos aumentando la tasa de propagación del virus. En el proceso de propagación de la enfermedad, el individuo susceptible se traslada a la población infectada $I(t)$.

$P(t)$ es el número o cantidad de patógenos presentes durante la interacción de los seres humanos en el tiempo $t$. La mayoría de los individuos infectados se recuperan y se trasladan a la población humana recuperada $\mathrm{R}(\mathrm{t})$.

El modelo compartimental se muestra en la Figura 1.

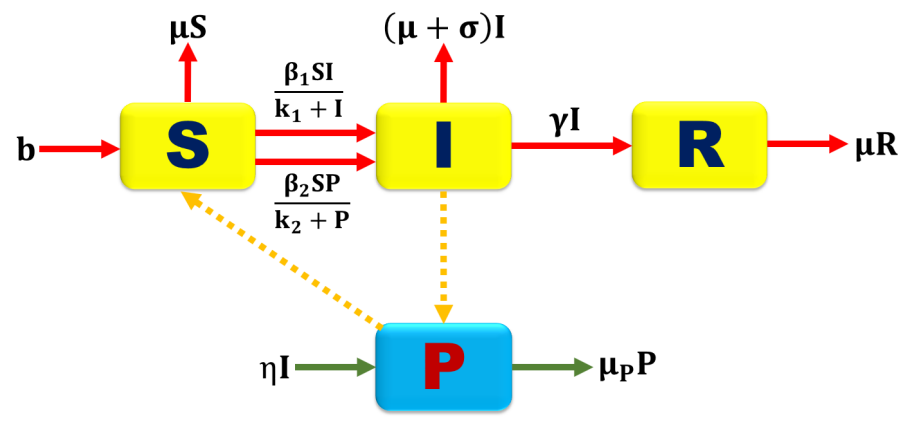

Figura 1: Esquema del modelo SIR-P

Ahora describiremos las ecuaciones del modelo matemático $S I R-P$, cuyo sistema de ecuaciones diferenciales ordinarias no lineales, esta representado de la siguiente forma:

$$
\begin{aligned}
\frac{d S}{d t} & =b-\frac{\beta_{1} S I}{k_{1}+I}-\frac{\beta_{2} S P}{k_{2}+P}-\mu S \\
\frac{d I}{d t} & =\frac{\beta_{1} S I}{k_{1}+I}+\frac{\beta_{2} S P}{k_{2}+P}-(\mu+\gamma+\sigma) I \\
\frac{d R}{d t} & =\gamma I-\mu R \\
\frac{d P}{d t} & =\eta I-\mu_{p} P
\end{aligned}
$$

Con los siguientes parámetros:

- $b$ : tasa de natalidad de la población humana.

- $\mu$ : tasa de mortalidad natural.

- $\beta_{1}$ : tasa de contagio por el contacto de un Infectado I(t).

- $\beta_{2}$ : tasa de contagio por el contacto del patógeno $\mathrm{P}(\mathrm{t})$, presente en el medio ambiente y superficies.

- $\gamma$ : tasa de recuperación.

- $k_{1}$ : proporción de interacción por contacto con un Infectado I(t)

- $k_{2}$ : proporción de interacción por contacto del Patógeno $\mathrm{P}(\mathrm{t})$ en el medio ambiente y superficies. 
- $\sigma$ : tasa de mortalidad por la enfermedad.

- $\eta$ : tasa de propagación del patógeno por los Infectados.

- $\mu_{p}$ : tasa de mortalidad del patógeno.

\subsection{Análisis de estabilidad}

En las dos primeras ecuaciones del sistema (3), observamos que el compartimento de los Recuperados $R(t)$ no aparece,por lo tanto por simplicidad podemos omitirlo, es decir

$$
R(t)=N(t)-S(t)-I(t)
$$

Así pues el análisis de nuestro problema se centrará en el siguiente modelo reducido:

$$
\begin{aligned}
\frac{d S}{d t} & =b-\frac{\beta_{1} I S}{k_{1}+I}-\frac{\beta_{2} P S}{k_{2}+P}-\mu S \\
\frac{d I}{d t} & =\frac{\beta_{1} I S}{k_{1}+I}+\frac{\beta_{2} P S}{k_{2}+P}-(\mu+\gamma+\sigma) I \\
\frac{d P}{d t} & =\eta I-\mu_{p} P
\end{aligned}
$$

Ahora para encontrar los puntos de equilibrio de nuestro modelo, procederemos a igualar a cero, a cada ecuación de (4) y resolver dicho sistema:

$$
\begin{array}{r}
b-\frac{\beta_{1} I S}{k_{1}+I}-\frac{\beta_{2} P S}{k_{2}+P}-\mu S=0 \\
\frac{\beta_{1} I S}{k_{1}+I}+\frac{\beta_{2} P S}{k_{2}+P}-(\mu+\gamma+\sigma) I=0 \\
\eta I-\mu_{p} P=0
\end{array}
$$

En el caso del punto de equilibrio libre de enfermedad esto ocurre cuando $I(t)=0$ y $P(t)=0$, es decir:

$$
b-\mu S=0 \Rightarrow S=\frac{b}{\mu}
$$

por lo cual nuestro punto de equilibrio libre de enfermedad es $E_{0}=\left(\frac{b}{\mu} ; 0 ; 0\right)$ para el modelo 4 .

\subsection{Positividad y acotación del modelo}

Teorema 3.1 Sea el conjunto

$$
\Omega=\left\{(S(t), I(t), P(t)) \in \mathbf{R}_{+}^{3} \cup\{0\}: S(t)+I(t) \leq \frac{b}{\mu} \wedge P(t) \leq \frac{\eta b}{\mu_{p} \mu}, t \geq\right\}
$$

es para el sistema (4) una región positivamente invariante.

Demostración. De nuestro sistema (4), veamos si es positivamente invariante:

- Considerando $N=S+I$ y sumando las dos primeras ecuaciones de nuestro sistema (4) 
obtenemos

$$
\begin{aligned}
\frac{d S}{d t}+\frac{d I}{d t} & =b-\mu S-(\mu+\gamma+\sigma) I \\
\frac{d N}{d t} & \leq b-\mu N \\
\frac{d}{d t}\left[N e^{\mu t}\right] & \leq b e^{\mu t} \\
\int_{0}^{t} \frac{d}{d \tau}\left[N e^{\mu \tau}\right] d \tau & \leq \int_{0}^{t} b e^{\mu \tau} d \tau \\
N(t) e^{\mu t}-N(0) & \leq \frac{b}{\mu} e^{\mu t}-\frac{b}{\mu} \\
N(t) & \leq \frac{b}{\mu}
\end{aligned}
$$

- Ahora veamos si es positivamente invariante con respecto a la población de los patógenos,es decir de la tercera ecuación de nuestro sistema (4)

$$
\begin{aligned}
\frac{d P}{d t} & =\eta I-\mu_{p} P \\
\frac{d}{d t}\left[P e^{\mu_{p} t}\right] & \leq \eta \frac{b}{\mu} e^{\mu_{p} t} \\
\int_{0}^{t} \frac{d}{d \tau}\left[P e^{\mu_{p} \tau}\right] d \tau & \leq \int_{0}^{t} \frac{b}{\mu} e^{\mu_{p} \tau} d \tau \\
P(t) e^{\mu_{p} t}-P(0) & \leq \frac{\eta b}{\mu \mu_{p}} e^{\mu_{p} t}-\frac{\eta b}{\mu \mu_{p}} \\
P(t) & \leq \frac{\eta b}{\mu \mu_{p}}
\end{aligned}
$$

\subsection{Número Básico de Reproducción $\left(\mathcal{R}_{0}\right)$}

Para nuestro modelo planteado, usaremos como herramienta para el cálculo del $\mathcal{R}_{0}$, la Matriz de la Próxima Generación [4]. Supongamos que $x=(I, P)^{T}$, luego del sistema (4), se tiene que:

$$
\frac{d x}{d t}=\mathcal{F}-\mathcal{V}
$$

Donde:

$$
\mathcal{F}=\left(\begin{array}{c}
\frac{\beta_{1} I S}{k_{1}+I}+\frac{\beta_{2} P S}{k_{2}+P} \\
0
\end{array}\right) \quad \mathcal{V}=\left(\begin{array}{c}
(\mu+\gamma+\sigma) I \\
-\eta I+\mu_{p} P
\end{array}\right)
$$

Teniendo en cuenta que $C=\mu+\gamma+\sigma$, obtenemos que:

$$
F=J\left[\mathcal{F}\left(E_{0}\right)\right]=\left(\begin{array}{cc}
\frac{\beta_{1} b}{\mu k_{1}} & \frac{\beta_{2} b}{\mu k_{2}} \\
0 & 0
\end{array}\right) \quad V=J\left[\mathcal{V}\left(E_{0}\right)\right]=\left(\begin{array}{cc}
C & 0 \\
-\eta & \mu_{p}
\end{array}\right)
$$

Ahora calculemos la inversa de $V$, es decir:

$$
V^{-1}=\left(\begin{array}{cc}
\frac{1}{C} & 0 \\
\frac{\eta}{\mu_{p} C} & \frac{1}{\mu_{p}}
\end{array}\right)
$$


Entonces la matriz de la próxima generación para el sistema (4) esta dado por :

$$
K=F V^{-1}=\left(\begin{array}{cc}
\frac{\beta_{1} b}{\mu C k_{1}}+\frac{\beta_{2} b \eta}{\mu \mu_{p} C k_{2}} & \frac{\beta_{2} b}{\mu \mu_{p} k_{2}} \\
0 & 0
\end{array}\right)
$$

Por lo tanto el radio espectral de la matriz $K=F V^{-1}$, es el número de reproducción básica, es decir $\mathcal{R}_{0}=\rho\left(F V^{-1}\right)$, entonces:

$$
\mathcal{R}_{0}=\frac{\beta_{1} b}{\mu C k_{1}}+\frac{\beta_{2} b \eta}{\mu \mu_{p} C k_{2}}
$$

Denotando:

$$
\mathcal{R}_{0}^{H}=\frac{\beta_{1} b}{\mu C k_{1}} \quad \wedge \quad \mathcal{R}_{0}^{P}=\frac{\beta_{2} b \eta}{\mu \mu_{p} C k_{2}}
$$

Entonces es preciso indicar que $\mathcal{R}_{0}^{H}$ esta vinculada a la transmisión directa y $\mathcal{R}_{0}^{P}$ esta relacionada a la transmisión indirecta.Si alguno de ellos es mayor que uno, entonces el número de reproducción básico $\mathcal{R}_{0}>1$, esto enfatiza el hecho de que para reducir la propagación del virus, se deben controlar todas las vías de transmisión.

\subsection{Existencia del punto de equilibrio endémico}

Para encontrar el punto de equilibrio endémico tenemos que resolver en función del sistema mencionado en (5), es decir

$$
\begin{aligned}
b-\frac{\beta_{1} I^{*} S^{*}}{k_{1}+I^{*}}-\frac{\beta_{2} P^{*} S^{*}}{k_{2}+P^{*}}-\mu S^{*} & =0 \\
\frac{\beta_{1} I^{*} S^{*}}{k_{1}+I^{*}}+\frac{\beta_{2} P^{*} S^{*}}{k_{2}+P^{*}}-(\mu+\gamma+\sigma) I^{*} & =0 \\
\eta I^{*}-\mu_{p} P^{*} & =0
\end{aligned}
$$

lo cual obtendremos respectivamente de la tercera ecuación y sumando la primera con la segunda la ecuación,lo siguiente :

$$
P^{*}=\frac{\eta I^{*}}{\mu_{p}} \quad \wedge \quad S^{*}=\frac{b-C I^{*}}{\mu}
$$

Claramente observamos que lo obtenido en 110$)$ está en función de $I^{*},\left(I^{*} \neq 0\right)$,ahora reemplazando en la primera ecuación,tendremos lo siguiente:

$$
\begin{aligned}
& 0=b-\frac{\beta_{1} I^{*} S^{*}}{k_{1}+I^{*}}-\frac{\beta_{2} P^{*} S^{*}}{k_{2}+P^{*}}-\mu S^{*} \\
& 0=\left(b-\mu S^{*}\right)\left(k_{1}+I^{*}\right)\left(k_{2}+P^{*}\right)-\beta_{1} I^{*} S^{*}\left(k_{2}+P^{*}\right)-\beta_{2} P^{*} S^{*}\left(k_{1}+I^{*}\right) \\
& 0=\mu C\left(k_{1}+I^{*}\right)\left(\mu_{p} k_{2}+\eta I^{*}\right)-\beta_{1}\left(b-C I^{*}\right)\left(\mu_{p} k_{2}+\eta I^{*}\right)-\beta_{2} \eta\left(b-C I^{*}\right)\left(k_{1}+I^{*}\right)
\end{aligned}
$$

Resolviendo,nos queda la siguiente ecuación de segundo grado:

$$
a_{1} I^{*^{2}}+a_{2} I^{*}+a_{3}=0
$$

Donde:

- $a_{1}=C \eta\left(\mu+\beta_{1}+\beta_{2}\right)$

- $a_{2}=\mu C \eta k_{1}\left(1-\mathcal{R}_{0}^{H}\right)+\mu \mu_{p} C k_{1} k_{2}\left(1-\mathcal{R}_{0}^{P}\right)+C\left(\beta_{1} \mu_{p} k_{2}+\beta_{2} \eta k_{1}\right)$

- $a_{3}=\mu \mu_{p} C k_{1} k_{2}\left(1-\mathcal{R}_{0}^{H}-\mathcal{R}_{0}^{P}\right)$ 
La solución de la ecuación (11) esta dado por

$$
I_{1 ; 2}^{*}=\frac{-a_{2} \pm \sqrt{a_{2}^{2}-4 a_{1} a_{3}}}{2 a_{1}}
$$

Analizando cada caso:

- Caso $1\left(\mathcal{R}_{0}<1\right)$. Tenemos que los coeficientes $a_{1}>0$ y $a_{3}>0$, además $a_{2}>0$ no tendrá raíces reales positivas, es decir el modelo no tiene punto de equilibrio endémico bajo estas condiciones.

- Caso $2\left(\mathcal{R}_{0}=1\right)$. Vemos que $a_{1}>0, a_{2}>0$ y además $a_{3}=\mu \mu_{p} C k_{1} k_{2}\left(1-\mathcal{R}_{0}\right)=0$,entonces para este caso tambien se comprueba que no tendrá raices reales positivas, es decir el modelo no tiene punto de equilibrio endémico bajo estas condiciones

- Caso $3\left(\mathcal{R}_{0}>1\right)$. Tenemos que $a_{1}>0$ y $a_{3}<0$, por lo tanto $a_{2}^{2}-4 a_{1} a_{3}>0$, es decir tiene raíces reales y diferentes, pero en este caso es indiferente el valor que tome el $a_{2}$ pues en cualquier caso se obtendría una única raíz real positiva. Es decir, el modelo (3) posee un único punto de equilibrio endémico.

\subsection{Estabilidad local}

Teorema 3.2 Si $\mathcal{R}_{0}<1$,entonces el modelo (4) para el caso del punto de equilibrio libre de enfermedad es localmente estable.

Demostración. Para $E_{0}$ la matriz jacobiana $\mathcal{J}\left(E_{0}\right)$ del sistema (4) esta dado por

$$
\mathcal{J}\left(E_{0}\right)=\left(\begin{array}{ccc}
-\mu & -\frac{\beta_{1} b}{\mu k_{1}} & -\frac{\beta_{2} b}{\mu k_{2}} \\
0 & \frac{\beta_{1} b}{\mu k_{1}}-C & \frac{\beta_{2} b}{\mu k_{2}} \\
0 & \eta & -\mu_{p}
\end{array}\right)
$$

Calculando ahora el polinomio característico asociado a $\mathcal{J}\left(E_{0}\right)$,obtenemos lo siguiente

$$
P(\lambda)=-(\lambda+\mu)\left[\lambda^{2}+\left(\frac{\mu C k_{1}-\beta_{1} b+\mu \mu_{p} k_{1}}{\mu k_{1}}\right) \lambda+\frac{\mu \mu_{p} C k_{1} k_{2}-\beta_{1} b \mu_{p} k_{2}-\beta_{2} b \eta k_{1}}{\mu k_{1} k_{2}}\right]
$$

Observamos que claramente $\lambda_{1}=-\mu<0$ es un autovalor del polinomio característico, además sabemos que para garantizar la estabilidad local de nuestro modelo dichos autovalores deben tener parte real no positiva, en el caso de los otros dos autovalores usaremos el criterio de RouthHurwitz [5], 6]; para determinar bajo que condiciones el tiene raices con parte real no positiva, lo cual obtendremos que

$$
\begin{array}{r}
\frac{\mu \mu_{p} C k_{1} k_{2} \beta_{1} b \mu_{p} k_{2}+\beta_{2} b \eta k_{1}}{\mu k_{1} k_{2}}>0 \Rightarrow \mu \mu_{p} C k_{1} k_{2}>\beta_{1} b \mu_{p} k_{2}+\beta_{2} b \eta k_{1} \\
\Rightarrow 1>\mathcal{R}_{0}^{H}+\mathcal{R}_{0}^{P} \Rightarrow \mathcal{R}_{0}<1
\end{array}
$$

Por lo cual se demuestra que $E_{0}$ es localmente estable.

Teorema $3.3 \mathrm{El}$ punto de equilibrio endémico $E_{1}^{*}\left(S^{*}, I^{*}, P^{*}\right)$ es localmente estable si satisfacen las siguientes desigualdades (13), (14) y (15):

$$
\frac{\beta_{1} S^{*} k_{1}}{\left(k_{1}+I^{*}\right)^{2}}<N_{1}
$$




$$
\begin{gathered}
\left.\frac{\beta_{1} S^{*} k_{1} \mu_{p} C}{\left(k_{1}+I^{*}\right)^{2}}+\left[\frac{\beta_{1} I^{*}}{k_{1}+I^{*}}+\frac{\beta_{2} P^{*}}{k_{2}+P^{*}}+\mu\right)\right]\left[\frac{\beta_{1} S^{*} k_{1}\left(\mu_{p}+C\right)}{\left(k_{1}+I^{*}\right)^{2}}+\mu_{p} C\right] \\
+\left[\mu+\mu_{p}+C+\frac{\beta_{1} I^{*}}{k_{1}+I^{*}}+\frac{\beta_{2} P^{*}}{k_{2}+P^{*}}\right]\left[\frac{\beta_{1} S^{*} k_{1}\left(\mu+\mu_{p}\right)}{\left(k_{1}+I^{*}\right)^{2}}+\frac{\beta_{2} S^{*} k_{2} \eta}{\left(k_{2}+P^{*}\right)^{2}}\right]<N_{2} \\
\frac{\beta_{1} S^{*} k_{1} \mu \mu_{p}}{\left(k_{1}+I^{*}\right)^{2}}+\frac{\beta_{2} S^{*} k_{2} \eta \mu}{\left(k_{2}+P^{*}\right)^{2}}<N_{3}
\end{gathered}
$$

Donde

$$
\begin{aligned}
N_{1} & =\mu+\mu_{p}+C+\frac{\beta_{1} I^{*}}{k_{1}+I^{*}}+\frac{\beta_{2} P^{*}}{k_{2}+P^{*}} \\
N_{2} & =\left[\mu+\mu_{p}+C+\frac{\beta_{1} I^{*}}{k_{1}+I^{*}}+\frac{\beta_{2} P^{*}}{k_{2}+P^{*}}\right]\left[\mu_{p} C+\left(\mu_{p}+C\right)\left(\frac{\beta_{1} I^{*}}{k_{1}+I^{*}}+\frac{\beta_{2} P^{*}}{k_{2}+P^{*}}+\mu\right)\right] \\
& +\frac{\beta_{1} S^{*} k_{1}}{\left(k_{1}+I^{*}\right)^{2}}\left[\frac{\beta_{1} S^{*} k_{1}\left(\mu+\mu_{p}\right)}{\left(k_{1}+I^{*}\right)^{2}}+\frac{\beta_{2} S^{*} k_{2} \eta}{\left(k_{2}+P^{*}\right)^{2}}\right]+\frac{\beta_{1} S^{*} k_{1} \mu \mu_{p}}{\left(k_{1}+I^{*}\right)^{2}}+\frac{\beta_{2} S^{*} k_{2} \mu \eta}{\left(k_{2}+P^{*}\right)^{2}} \\
N_{3} & =\left(\frac{\beta_{1} I^{*}}{k_{1}+I^{*}}+\frac{\beta_{2} P^{*}}{k_{2}+P^{*}}+\mu\right) \mu_{p} C
\end{aligned}
$$

Demostración. Calculando ahora el polinomio característico asociado a $\mathcal{J}\left(E_{1}\right)$,obtenemos lo siguiente

$$
P(\lambda)=\operatorname{det}\left(\mathcal{J}\left(E_{1}\right)-\lambda I\right)=\left|\begin{array}{ccc}
-\mu-\frac{\beta_{1} I^{*}}{k_{1}+I^{*}}-\frac{\beta_{2} P^{*}}{k_{2}+P^{*}}-\lambda & -\frac{\beta_{1} S^{*} k_{1}}{\left(k_{1}+I^{*}\right)^{2}} & -\frac{\beta_{2} S^{*} k_{2}}{\left(k_{2}+P^{*}\right)^{2}} \\
\frac{\beta_{1} I^{*}}{k_{1}+I^{*}}+\frac{\beta_{2} P^{*}}{k_{2}+P^{*}} & \frac{\beta_{1} S^{*} k_{1}}{\left(k_{1}+I^{*}\right)^{2}}-C-\lambda & \frac{\beta_{2} S^{*} k_{2}}{\left(k_{2}+P^{*}\right)^{2}} \\
0 & \eta & -\mu_{p}-\lambda
\end{array}\right|
$$

Donde:

$$
Z_{1}=\frac{\beta_{1} I^{*}}{k_{1}+I^{*}}+\frac{\beta_{2} P^{*}}{k_{1}+P^{*}} \wedge Z_{2}=\frac{\beta_{1} S^{*} k_{1}}{\left(k_{1}+I^{*}\right)^{2}} \quad \wedge \quad Z_{3}=\frac{\beta_{2} S^{*} k_{2}}{\left(k_{2}+P^{*}\right)^{2}}
$$

Realizando los cálculos respectivos, obtenemos

$$
P(\lambda)=\lambda^{3}+\alpha_{1} \lambda^{2}+\alpha_{2} \lambda+\alpha_{3}=0
$$

Donde:

$$
\begin{aligned}
& \alpha_{1}=\left(\mu+\mu_{p}+C+Z_{1}\right)-Z_{2} \\
& \alpha_{2}=\left[\mu_{p} C+\left(\mu_{p}+C\right)\left(Z_{1}+\mu\right)\right]-\left[Z_{2}\left(\mu+\mu_{p}\right)+Z_{3} \eta\right] \\
& \alpha_{3}=\mu_{p} C\left(Z_{1}+\mu\right)-\left[\mu \mu_{p} Z_{2}+\eta \mu Z_{3}\right]
\end{aligned}
$$

Por el criterio de Routh Hurwitz, analizaremos la estabilidad de la ecuación (16) para asegurarnos que sus raíces tengan parte real negativa. 


\begin{tabular}{c|cc}
$\lambda^{3}$ & 1 & $\alpha_{2}$ \\
$\lambda^{2}$ & $\alpha_{1}$ & $\alpha_{3}$ \\
$\lambda^{1}$ & $b_{1}$ & $b_{2}$ \\
$\lambda^{0}$ & $c_{1}$ &
\end{tabular}

en la primera columna del arreglo de Routh debemos asegurarnos que no haya cambios de signos es decir debemos asegurar que $\alpha_{1}>0, b_{1}>0$ y $c_{1}>0$,donde:

$$
b_{1}=\frac{\alpha_{1} \alpha_{2}-\alpha_{3}}{\alpha_{1}} \quad \wedge \quad c_{1}=\alpha_{3}
$$

Bajo estas condiciones se demuestra que el punto de equilibrio endémico $E_{1}=\left(S^{*}, I^{*}, P^{*}\right)$ es localmente estable si satisfacen las ecuaciones $(13),(14)$ y $(15)$.

\subsection{Estabilidad Global del punto de equilibrio $E_{0}$}

Teorema 3.4 Si $\mathcal{R}_{0} \leq 1$, entonces el punto de equilibrio libre de enfermedad $E_{0}$ del modelo (3) es globalmente estable.

Demostración. Consideremos en primer lugar las matrices $F$ y $V$ mencionadas en la ecuación (7), además recordar que la matriz inversa esta dado por (8). También podemos observar que $F \geq 0$ y $V^{-1} \geq 0$. Además se tiene que $x^{T}=(I, P)$ y $y^{T}=(S, R)$, lo cual nos permite expresar los compartimentos de la enfermedad de la siguiente manera

$$
x^{\prime}=(F-V) x-f(x, y)
$$

Donde definimos $f(x, y)$ de la siguiente forma

$$
\begin{aligned}
f(x, y) & =(F-V) x-\mathcal{F}(x, y)+\mathcal{V}(x, y) \\
& =\left(\begin{array}{cc}
-C+\frac{\beta_{1} b}{\mu k_{1}} & \frac{\beta_{2} b}{\mu k_{2}} \\
\eta & -\mu_{p}
\end{array}\right)\left(\begin{array}{c}
I \\
P
\end{array}\right)-\left(\frac{\beta_{1} I S}{k_{1}+I}+\frac{\beta_{2} P S}{k_{2}+P}\right)+\left(\begin{array}{c}
C I \\
0 \\
-\eta I+\mu_{p} P
\end{array}\right) \\
& =\left(\begin{array}{cc}
\left.\beta_{1} I\left[\frac{b}{\mu k_{1}}-\frac{S}{K_{1}+I}\right]+\beta_{2} P\left[\frac{b}{\mu k_{2}}-\frac{S}{K_{2}+P}\right]\right) \\
0
\end{array}\right.
\end{aligned}
$$

Se sabe que $S \leq \frac{b}{\mu}$, por lo cual se deduce que

$$
\frac{b}{\mu k_{1}}-\frac{S}{K_{1}+I}=\frac{(b-S \mu) k_{1}+b I}{\mu K_{1}\left(K_{1}+I\right)} \geq 0 \quad \wedge \quad \frac{b}{\mu k_{2}}-\frac{S}{K_{2}+P}=\frac{(b-S \mu) k+b P}{\mu K_{2}\left(K_{1}+P\right)} \geq 0
$$

Con lo cual podemos ver claramente que $f(x, y) \geq 0$. Con lo cual podemos construir una función de Lyapunov como nos hace referencia el teorema 2.1 en [7], es decir

$$
Q(t)=\omega^{T} V^{-1} x(t)
$$

Donde $\omega$ es el autovector a la izquierda de la matriz no negativa $V^{-1} F$ con autovalor $\mathcal{R}_{0}$, donde haciendo el cálculo respectivo encontramos que

$$
\omega^{T}=\left[\begin{array}{ll}
\frac{\beta_{1} k_{2}}{\beta_{2} k_{1}} & 1
\end{array}\right]
$$

Derivando $Q$ de la ecuación (17), obtenemos

$$
\begin{aligned}
Q^{\prime}(t) & =\omega^{T} V^{-1} x^{\prime}(t) \\
& =\omega^{T} V^{-1}(F-V) x(t)-\omega^{T} V^{-1} f(x, y) \\
& \leq \omega^{T}\left(V^{-1} F-I_{2}\right) x(t)
\end{aligned}
$$


Donde

$$
\begin{aligned}
& \omega^{T}\left(V^{-1} F-I_{2}\right)=\left[\begin{array}{ll}
\frac{\beta_{1} k_{2}}{\beta_{2} k_{1}} & 1
\end{array}\right]\left(\begin{array}{cc}
\mathcal{R}^{H}-1 & \frac{\mu_{p}}{\eta} \mathcal{R}^{P} \\
\frac{\eta}{\mu_{p}} \mathcal{R}^{H} & \mathcal{R}^{P}-1
\end{array}\right) \\
& =\left(\mathcal{R}_{0}-1\right)\left[\begin{array}{ll}
\frac{\beta_{1} k_{2}}{\beta_{2} k_{1}} & 1
\end{array}\right]
\end{aligned}
$$

De esta última expresión podemos deducir que

$$
\Rightarrow Q^{\prime} \leq\left(\mathcal{R}_{0}-1\right) \omega^{T} x(t)
$$

tal que $I_{2}$ es la matriz identidad de orden 2. Para demostrar que el punto de equilibrio libre de infección $E_{0}$ es globalmente estable, se necesita demostrar que $\mathcal{R}_{0} \leq 1$ implica que $x=0$.

Si $\mathcal{R}_{0}<1$,es evidente que $Q^{\prime} \leq 0$, pero en el caso particular que $Q^{\prime}=0$ esto implica que $x=0$ por lo tanto $f(0, y)=0$. Si $\mathcal{R}_{0}=1$, en el caso que $Q^{\prime}=0$ implica también que $x=0$.

Además por el principio de invarianza de LaSalle, teniendo en cuenta que $E_{0}$ es el mayor conjunto invariante $\Omega_{0}=\left\{(S, I, R, P) \in \Omega, Q^{\prime}=0\right\}$, se puede concluir que $E_{0}$ es un punto atractor, y por lo tanto es globalmente estable cuando $\mathcal{R}_{0} \leq 1$.

\section{Resultados}

Ahora mostraremos la simulación numérica del modelo planteado (3), haciendo uso del lenguaje de Python,para obtener gráficos que nos ayuden a interpretar el modelo.Los parámetros del Cuadro (1) en su mayor parte,han sido tomados de un artículo de Mwalili [8] y los valores de los parámetros $k_{1}$ y $k_{2}$ se han asumido, teniendo en cuenta que se ajuste al modelo con respecto a su $\mathcal{R}_{0}$, además hemos tomado como datos iniciales de cada compartimentos de nuestro modelo, los siguientes valores: $S(0)=93000, I(0)=50, R(0)=0, P(0)=500$, en este caso inicial el valor aproximado del $\mathcal{R}_{0}=0,615$ bajo los parámetros mencionados.

\begin{tabular}{||c||c||c||}
\hline \multicolumn{1}{|c||}{ Nombre del parámetro } & Símbolo & Valor \\
\hline \hline Tasa de Natalidad de la población humana & $\mathrm{b}$ & 0.00018 tiempo $^{-1}$ \\
Tasa de mortalidad natural de la población humana & $\mu$ & 0.000046 tiempo $^{-1}$ \\
Tasa de contagio de I(t) a S(t) & $\beta_{1}$ & 0.0115 \\
Tasa de contagio de P(t) a S(t) & $\beta_{2}$ & 0.00414 \\
Tasa de recuperación & $\gamma$ & 0.05 tiempo $^{-1}$ \\
Proporción de interacción por contacto con un I(t) & $k_{1}$ & 2 \\
Proporción de interaccipon por contacto con P(t) & $k_{2}$ & 1 \\
Tasa de mortalidad por la enfermedad & $\sigma$ & 0.0018 \\
Tasa de propagación del P(t) en el medio ambiente & $\eta$ & 0.1 tiempo $^{-1}$ \\
Tasa de mortalidad del patógeno & $\mu_{p}$ & 0.1724 tiempo $^{-1}$ \\
\hline
\end{tabular}

Cuadro 1: Parámetros del modelo.

En este primer escenario (Figura2) de la simulación epidemiológica,el cual se está trabajando con respecto a los parámetros iniciales del Cuadro (1), se puede observar que el $\mathcal{R}_{0}^{H}=0,434$ y el $\mathcal{R}_{0}^{P}=0,1812$,es decir $\mathcal{R}_{0}=0,6152$,podemos también indicar que los humanos susceptibles y los humanos recuperados bajan y suben moderamente con el transcurso del tiempo respectivamente,además los humanos infectados llegan a su mayor cantidad el día 33 con un total de 16700 individuos,luego de esto comienzan a descender hasta desaparecer con el transcurso del tiempo. 


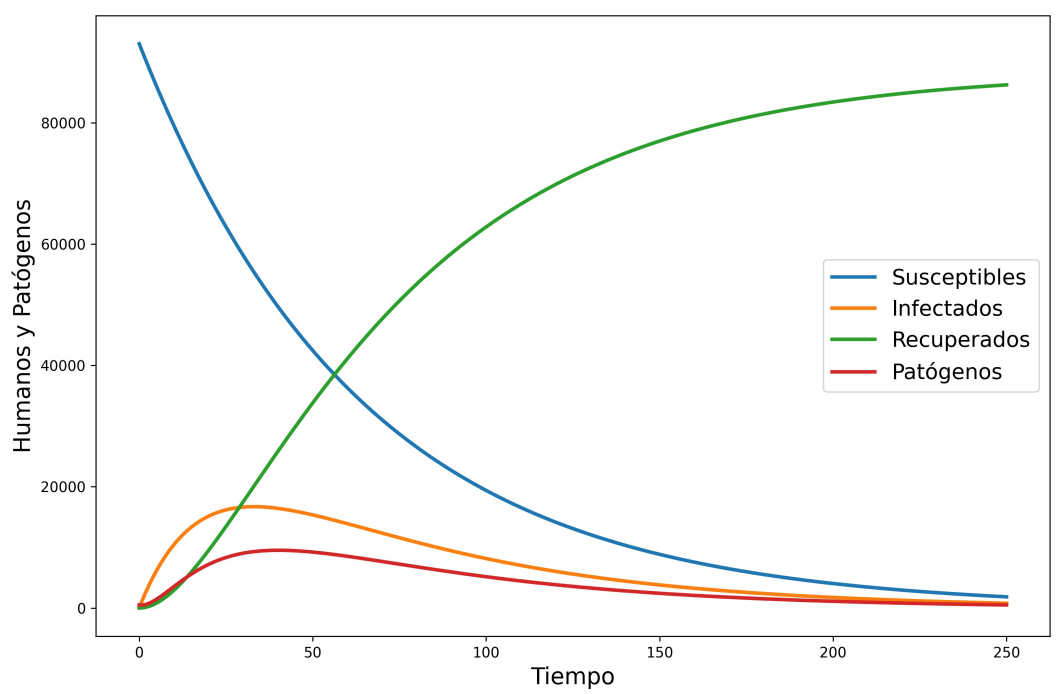

Figura 2: Simulación de la población humana con el patógeno $\left(\mathcal{R}_{0}=0,6152\right)$

En este segundo escenario (Figura33), como se ha mencionado se ha realizado un a modificación en las tasas de contagio $\beta_{1}=0,1$ y $\beta_{2}=0,05$ con respecto a los parámetros iniciales del Cuadro (1), se puede verificar que en el caso del $\mathcal{R}_{0}^{H}=3,7737$ y el $\mathcal{R}_{0}^{P}=2,1819$, es decir $\mathcal{R}_{0}=5,9626$,se puede observar que el número de susceptible decae considerablemente en los primeros 20 días,además en el caso de los humanos infectados aumenta rápidamente en los primeros días,siendo su máximo valor en el día 11 con 53049 individuos,luego de esto comienza a descender rápidamente.

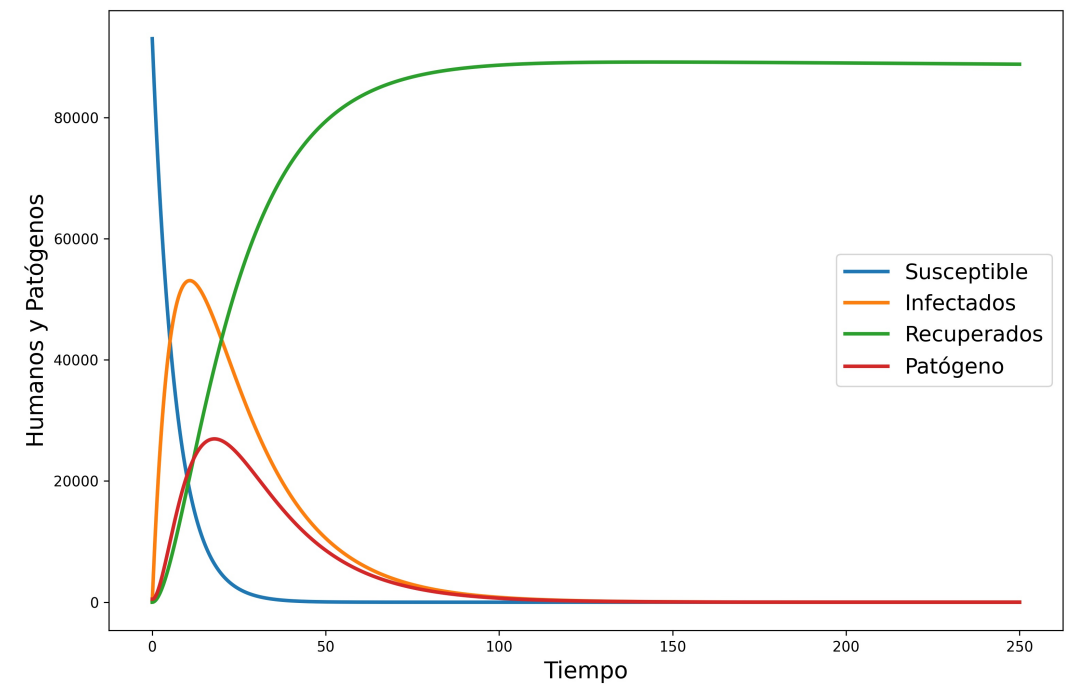

Figura 3: Simulación de la población humana con el patógeno $\left(\mathcal{R}_{0}=5,9626\right)$

En las siguientes gráficas (Figura 4 5 6 67) se pueden observar mejor cada compartimento (Susceptibles,Infectados,Recuperados y Patógenos) la simulación de nuestro modelo epidemiológico con las variaciones de tasas de contagio que se planteo. 


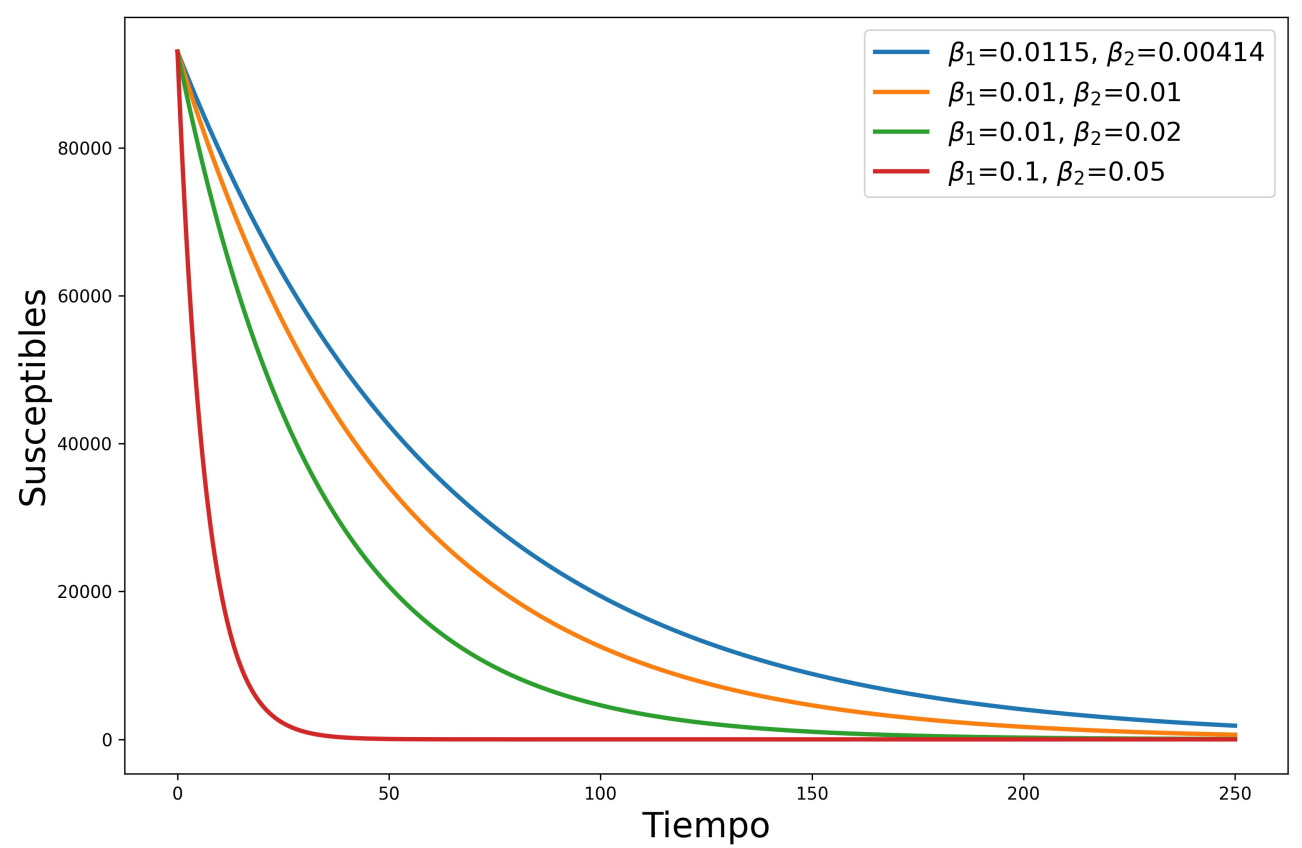

Figura 4: Población de Susceptibles variando las tasas de contagios

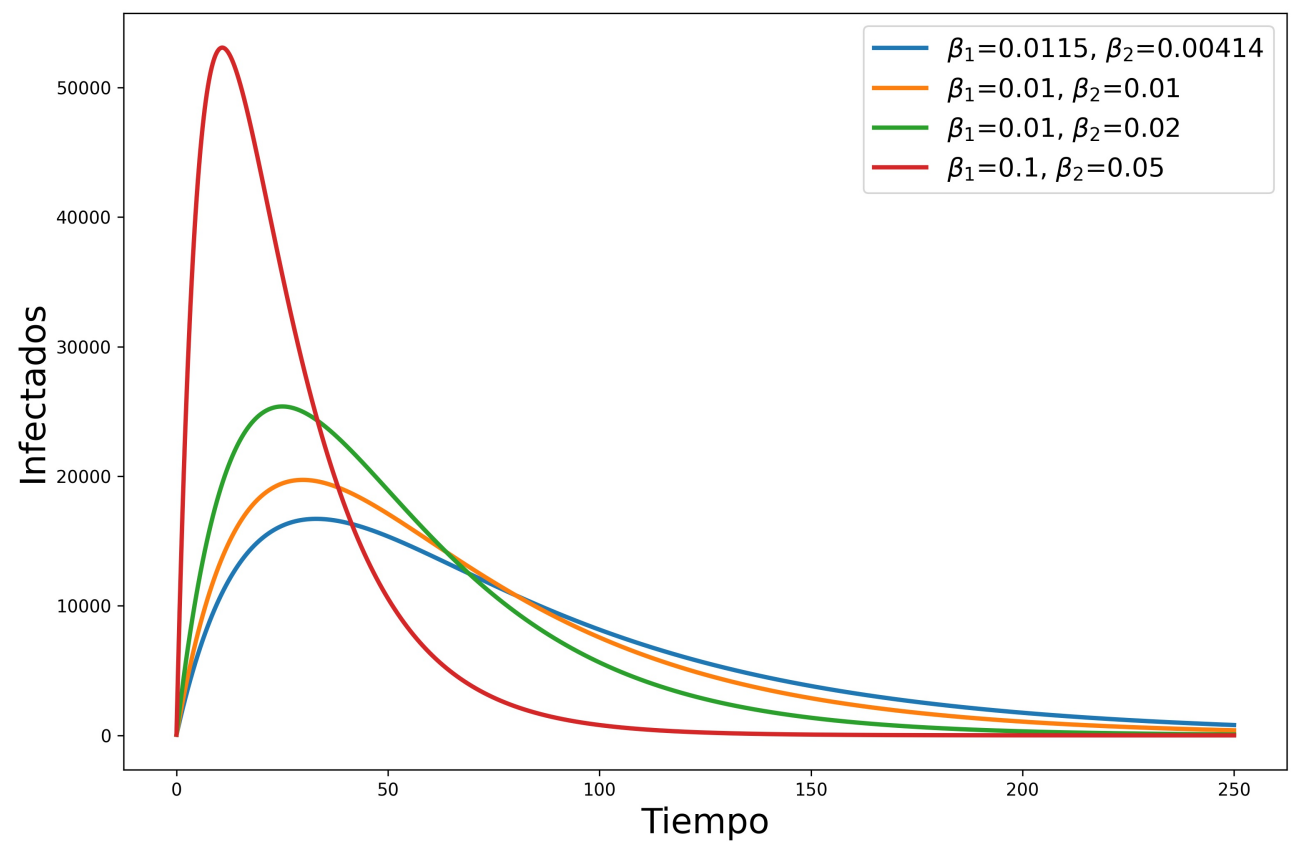

Figura 5: Población de Infectados variando las tasas de contagios. 


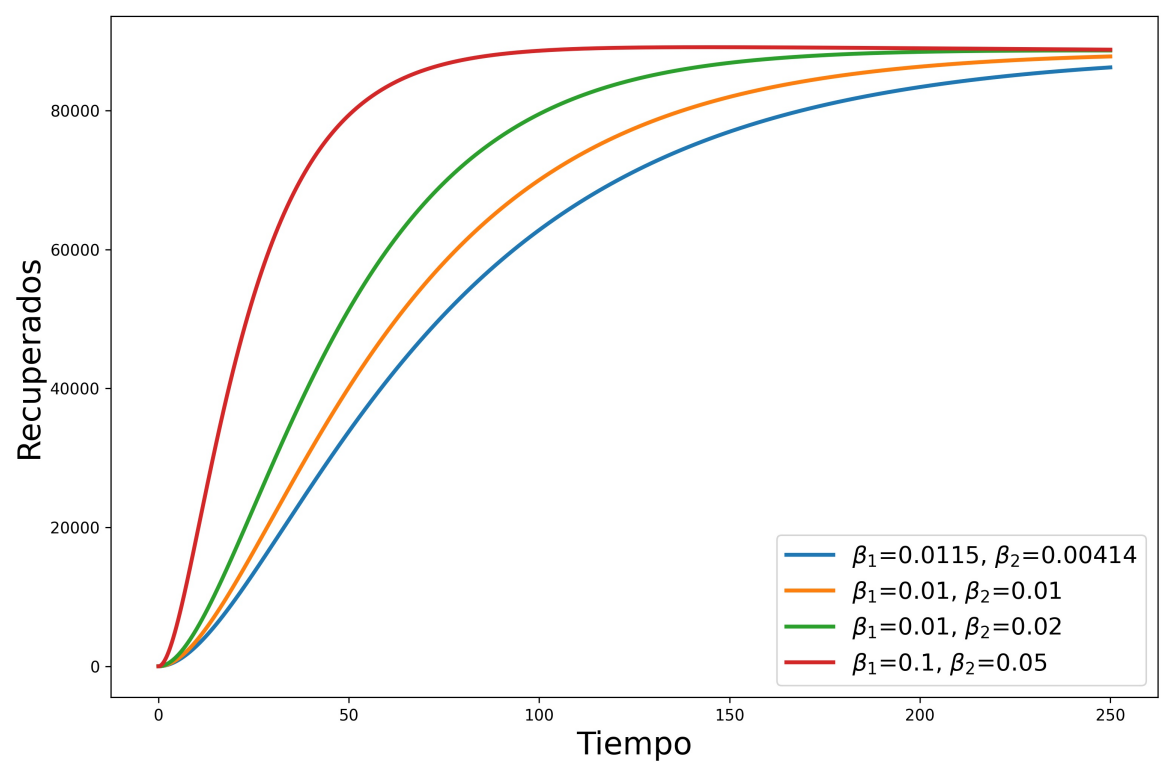

Figura 6: Población de Recuperados variando las tasas de contagios.

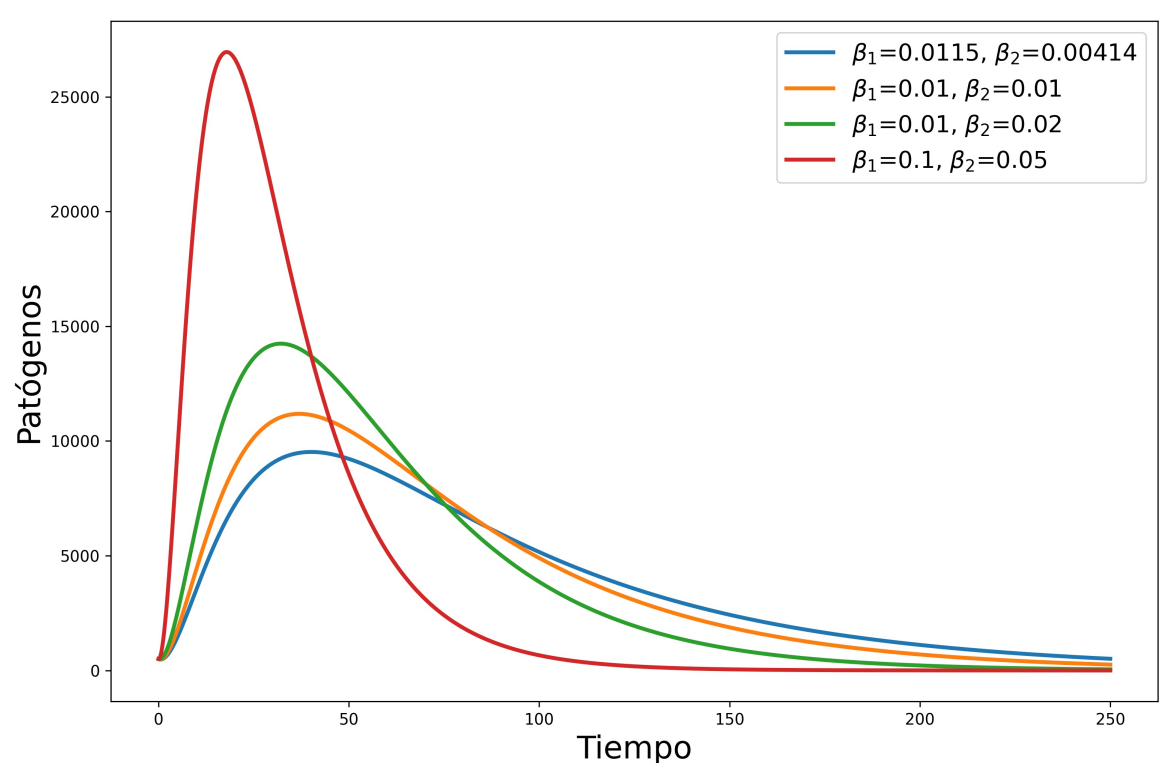

Figura 7: Población de Patógenos variando las tasas de contagios.

A parte de estas simulaciones, se quizó observar las consecuencias de la variación de la tasa de recuperación con respecto a los humanos recuperados como se muestra en la Figura 8 y también la variación de la tasa de propagación del virus en el medio ambiente,la cual se observa que si hay un cierto impacto en el crecimiento poblacional del patógeno, mientras aumenta dicha tasa como se verifica en la Figura 9. 


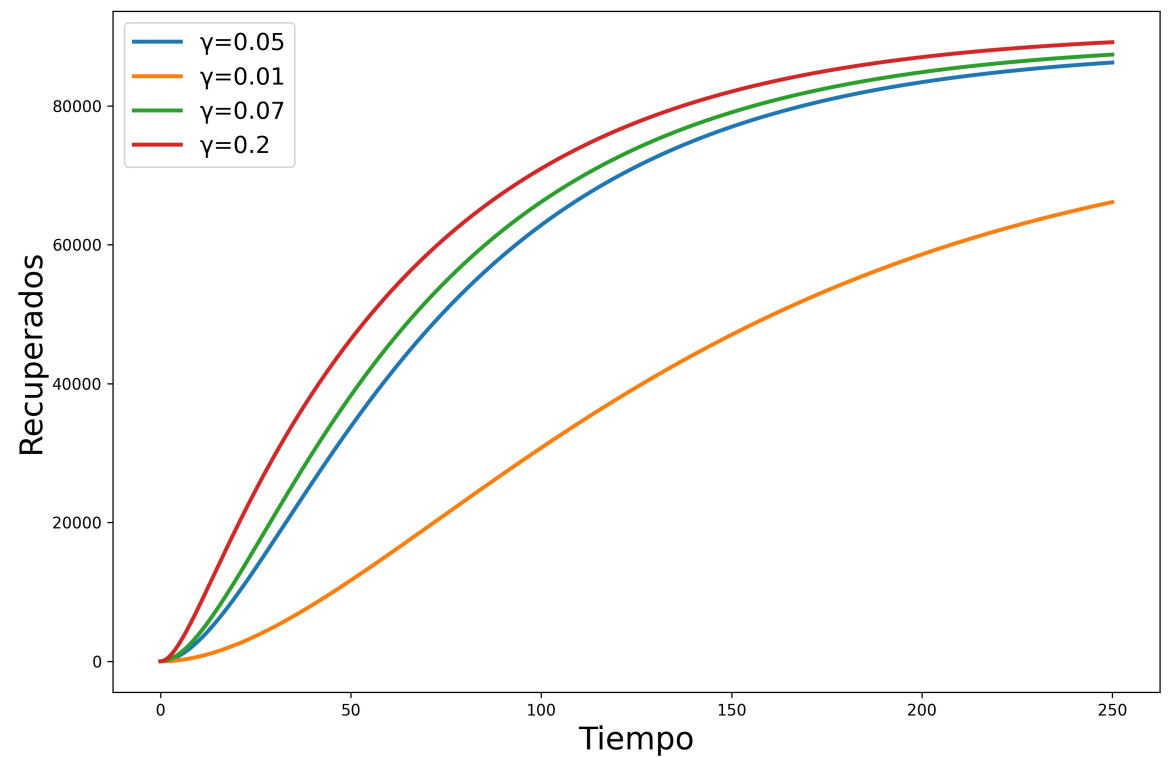

Figura 8: Población de Recuperados variando las tasas de recuperación

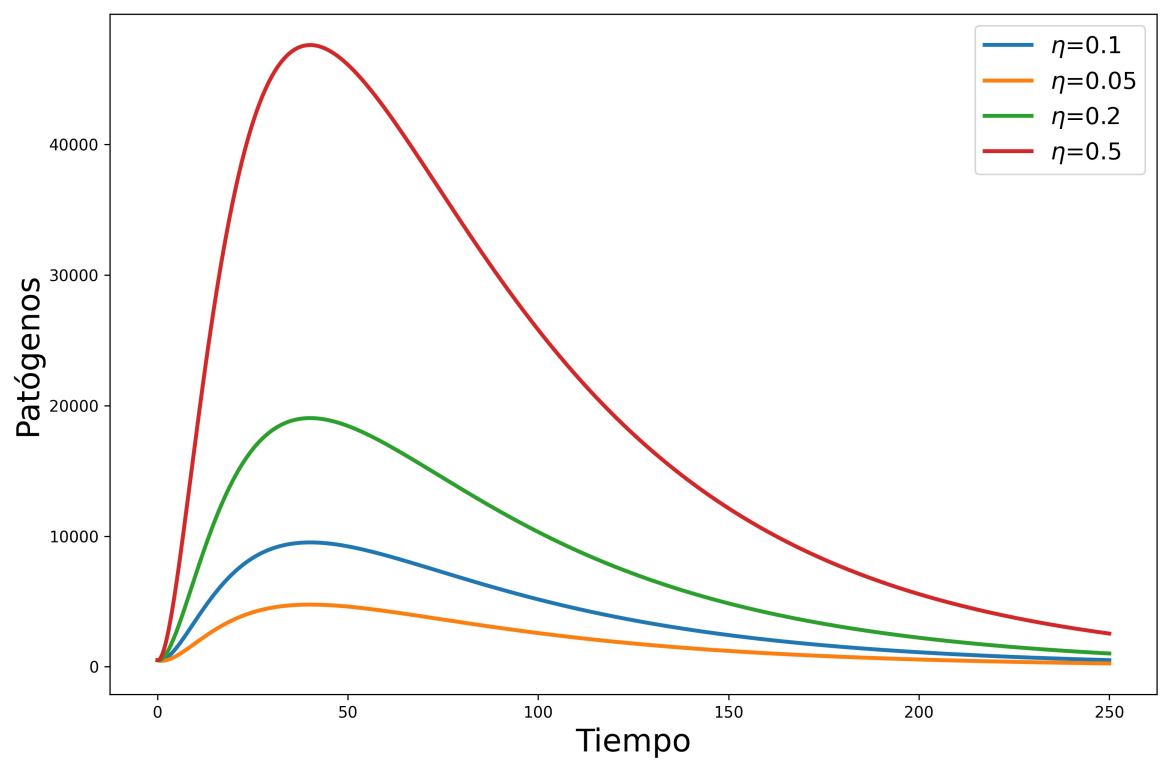

Figura 9: Población de Patógenos variando la tasa de propagación

\section{Discusión}

Es evidente que solamente se esta tomando un modelo básico en el cual se puede ampliar los compartimentos de los humanos ( Infectados sintomáticos e Infectados asisntomáticos) y además de poder considerar otros parámetros que se ajusten al modelo, como en el trabajo de Mwalili [8] y otros más, el cual hace un estudio del $\mathcal{R}_{0}$ y algunas simulaciones, donde se debe profundizar la estabilidad global y otros aspectos. 


\section{Conclusión}

1. El análisis de estabilidad es primordial para el estudio de nuestro modelo matemático, en esta investigación se ha realizado el análisis de estabilidad del punto libre de infección tanto local como global, y en el caso del punto endémico se ha realizado la estabilidad local.

2. En este trabajo se ha variado los parámetros para ver el impacto en nuestro modelo epidemiológico, lo cual en un inicio se ha considerado como referencias un estudio realizado en un artículo 8 .

3. Las medidas de prevención, como el distanciamiento social,el uso de mascarillas o el lavado de manos toman relevancia en nuestro modelo, pues ayuda a que no se propague con intensidad el virus, por lo cual ayudará que esta enfermedad emergente se mitigue durante el transcurso de un cierto tiempo.

\section{Referencias bibliográficas}

[1] Kermack, W. O., \& McKendrick, A. G. (1927). "A contribution to the mathematical theory of epidemics". Proceedings of the royal society of london. Series A, Containing papers of a mathematical and physical character, 115(772), 700-721.

[2] López-Cruz, R. (2006). "Structured SI epidemic models with applications to HIV epidemic ". Arizona State University.

[3] Khalil, H. K.,\& Grizzle, J. W. (2002). "Nonlinear Systems". Upper Saddle River, NJ: Prentice Hall.

[4] Van den Driessche, P., \& Watmough, J. (2002). "Reproduction numbers and sub-threshold endemic equilibria for compartmental models of disease transmission". Mathematical biosciences, 180(1-2), 29-48.

[5] Allen, L. J. (2007). "Introduction to mathematical biology ". Pearson/Prentice Hall.

[6] Bolton, W., \& Ramírez, F. J. R. (2001). "Ingeniería de control"(pp. 1-3). Mexico: Alfaomega.

[7] Shuai, Z., \& van den Driessche, P. (2013). "Global stability of infectious disease models using Lyapunov functions". SIAM Journal on Applied Mathematics, 73(4), 1513-1532.

[8] Mwalili, S., Kimathi, M., Ojiambo, V., Gathungu, D., \& Mbogo, R. (2020). "SEIR model for COVID-19 dynamics incorporating the environment and social distancing". BMC Research Notes, 13(1), 1-5. 